\section{PP-05 HEPATITIS E OUTBREAKS IN URBAN AREAS: CHALLENGES FOR INTERSECTORAL ACTION}

Samir Garg, Shikha Gupta, K Rizu. SHRC - State Health Resource Centre, Raipur (Chhattisgarh), India

\subsection{6/bmjgh-2016-EPHPabstracts.56}

Background An outbreak of hepatitis E in Raipur, capital of Chhattisgarh, was reported by press in April 2014. Outbreaks of hepatitis were also reported from the towns of Durg and Bhilai at the same time. In the neighbouring state of Orissa, several towns witnessed hepatitis outbreaks and the High Court of Orissa also intervened after deaths were reported.

Methods We conducted a study in Raipur to understand both outbreak and response. Data were collected from government laboratories, community health workers (CHWs, known as Mitanins) and 600 clinically confirmed hepatitis patients. Supervisors of CHWs were interviewed to understand the perspective of the cadre responsible for health education and mobilising referrals at community level. Maps of affected areas were drawn with help of CHWs and community leaders. Given the faeco-oral transmission of hepatitis E, maps covered drinking water sources, patient locations and dates of symptoms appearing. Water testing of various types of sources was done by CHWs using hydrogen sulfide kits. Contaminated sources were plotted on a city map.

Findings The Medical College Raipur tested 264 patients with jaundice symptoms for hepatitis E (blood serum ELISA) and hepatitis A, confirming 114 hepatitis $\mathrm{E}$ and three hepatitis A cases. CHWs reported 2,070 cases of hepatitis (including 74 pregnant women) and 32 deaths (including eight of pregnant women).

The media started covering the outbreak after two deaths were reported from an upper-class locality in April 2014. According to the data collected from CHWs, the outbreak started as early as December 2013 when around 30 persons from an impoverished slum locality showed jaundice. An NGO did water testing of 32 households (using hydrogen sulfide kits) in the mentioned slum in January 2014 and found that 17 of the households were drinking water presenting faecal contamination. Later CHWs also reported a large swine population in the same slum area.

According to survey conducted by the Mitanins, around 30\% of hepatitis cases had happened between December 2013 and
March 2014, though the departments started adressing Hepatitis only after mid April. Most of the cases were in the ago group above 14 years; the age group 20-49 years accounted for $60 \%$ of the cases; $30 \%$ of the water sources contributed to $90 \%$ of the caseload.

Water testing by Mitanins at 800 points across the city showed that $53 \%$ of sources including big pumping stations, hand pumps and the alternative water supply tankers presented faecal contamination. Another outbreak was experienced from January 2015 onwards, though in different areas. A repeat round of water testing was done by CHWs in April 2015, highlighting the spread of contamination. Media attention got shifted to swine flu outbreak in 2015 and on-going hepatitis outbreaks were ignored.

Discussion \& recommendations Hepatitis E outbreaks happened because conditions were conducive to high faeco-oral transmission. Leakages in drinking water pipelines and ingress of sewage into them took place and pumping stations were close to sewage in some areas. Swine population could have acted as reservoir.

Civic administration swung into action late April after media uproar on upper-class deaths. It organised daily reviews, medical camps and chlorine-tablet distribution. Poorer habitations were still largely ignored as upper-class areas dominated their attention. There was undue emphasis on hospitalisation of all patients and inadequate attention on managing dehydration. The actions were guided by the imperative to be seen as 'doing something'.

The civic administration was reluctant to accept that drinking water supplied by it was contaminated. Intersectoral action encountered severe governance issues with blame game between the health department and municipal bodies. There was ignorance in departments about existing protocols on water testing. Even the use of hydrogen sulfide kits was unknown, though eventually CHWs were equipped by the health department to conduct testing. The response of media reflected deep socioeconomic inequity.

Hepatitis outbreaks in urban areas need to be recognised as a critical public health problem demanding urgent intersectoral action. Governance reforms are needed to allow search and implementation of technical solutions by departments to prevent and contain contamination of drinking water supplies.

No competing interest. 